Palavras chave: Correlação de Spearman Fenofases Variáveis climáticas

Histórico: Recebido 21/08/2013 Aceito $01 / 12 / 2015$

Keywords: Spearman correlation Phenophases Climate variables

Correspondência: talitageorocha@yahoo.com.br
Talita Geovanna Fernandes Rocha', Richeliel Albert Rodrigues Silva', Eduarda Ximenes Dantas', Fábio de Almeida Vieira'

\title{
FENOLOGIA DA Copernicia prunifera (ARECACEAE) EM UMA ÁREA DE CAATINGA DO RIO GRANDE DO NORTE
}

RESUMO: O objetivo do presente trabalho foi avaliar a fenologia vegetativa e reprodutiva da Copernicia prunifera (carnaúba) em população natural, correlacionando-a com as variáveis climáticas. $O$ estudo foi realizado em uma área nativa, localizada no Campus da Unidade Acadêmica Especializada em Ciências Agrárias da UFRN, no município de Macaíba, RN. As avaliações foram feitas entre maio de 2010 e dezembro de 2012, totalizando 67 observações quinzenais das fenofases vegetativas e reprodutivas. As relações entre a ocorrência de cada fenofase e as variáveis climáticas (temperatura do ar, precipitação, umidade relativa, velocidade do vento e radiação) foram testadas por meio da correlação de Spearman, realizada com os dados climáticos da primeira $\left(r_{S_{1}}\right)$, segunda $\left(r_{52}\right)$, terceira $\left(r_{53}\right)$ e quarta $\left(r_{54}\right)$ quinzena anteriores ao evento fenológico. Os indivíduos de $C$. prunifera têm os eventos vegetativos assincrônicos, com perda de folhas e emissão de folhas novas durante todo o tempo. A floração é subanual, ou seja, ocorre mais de uma vez ao ano. Há relação positiva entre a intensidade de frutos imaturos e o período de maior temperatura, com atraso de três $\left(r_{s 3}=0,26 I ; P<0,005\right)$ a quatro quinzenas $\left(r_{s 4}=0,276 ; P<0,005\right)$ do evento fenológico. Foram observadas também correlações positivas e significativas entre a temperatura e a intensidade e número de árvores com frutos maduros. Adicionalmente, a intensidade de dispersão dos frutos maduros ocorre em períodos de menor umidade relativa do $\operatorname{ar}\left(r_{s 4}=-0,299 ; P<0,005\right)$. Assim, supõe-se que a espécie estudada foi influenciada por aspectos da morfologia e fisiologia, com os fatores ambientais interferindo secundariamente.

\section{PHENOLOGY OF Copernicia prunifera (ARECACEAE) IN A CAATINGA AREA OF RIO GRANDE DO NORTE}

ABSTRACT: The objective of this study was to evaluate the vegetative and reproductive phenology of Copernicia prunifera (carnauba wax) in natural populations correlated with climate variables. The study was conducted in a native area, located in the Campus of Academic Unit Specialized in Agricultural Sciences of UFRN in the municipality of Macaíba, RN. The evaluations were made between May 2010 and December 2012, totaling 67 fortnightly observations of vegetative phenophases and reproductive. The relationship between the occurrence of each phenophase and climate variables (air temperature, precipitation, relative humidity, wind speed and radiation) were tested by Spearman correlation, performed with the climate data of the first $\left(r_{s 1}\right)$, second $\left(r_{s 2}\right)$, third $\left(r_{s 3}\right)$ and fourth $\left(r_{s 4}\right)$ phenological fortnight prior to the event. Individuals of $C$. prunifera are asynchronous with leaf loss and emission of new leaves all the time. Flowering is subanual, i.e. occurs more than once a year. There is a positive relationship between the intensity of unripe fruits and higher temperature with delay of three $\left(r_{s 3}=0.26 I ; P<0.005\right)$ to four fortnights $\left(r_{54}=0.276 ; P<0.005\right)$ to the phenological event. We observed also significant positive correlations between temperature and number of trees with ripe fruits. Thus, it is assumed that the species studied was influenced by aspects of morphology and physiology, with environmental factors interfering secondarily. 


\section{INTRODUÇÃO}

Estudos fenológicos visam a compreensão do comportamento dos vegetais em correlação com as mudanças no ambiente biótico e abiótico, abrangendo os padrões estacionais de floração, frutificação e mudança foliar (VILELA et al., 2008). Desse modo, estudos que visam à caracterização das fenofases vegetais são importantes para a compreensão da dinâmica das populações de plantas, funcionando como indicadores das respostas das plantas às condições climáticas e edáficas locais (VIEIRA \& CARVALHO, 2009).

Os eventos de floração são determinantes para o sucesso reprodutivo das populações e podem influenciar na atração de polinizadores e no sucesso da frutificação (VILELA et al. 2008). A floração sincronizada facilita ○ fluxo de pólen e a reprodução cruzada (HALL et al., 1996). Assim, a biologia reprodutiva dos vegetais influencia a estrutura, o funcionamento e a regeneração das comunidades vegetais. Nos trópicos, fatores climáticos e interações entre espécies estão frequentemente associados com a sazonalidade dos eventos reprodutivos das plantas (CAMARGORICALDE et al., 2004).

Importante ressaltar ainda que algumas espécies têm uma fenofase ou fase fenológica de maior interesse para exploração e uso comercial. No caso da carnaúba está relacionada à emissão de folhas, pela estreita relação com o produto final de maior importância, o pó cerífero obtido das folhas. Além disso, os padrões de brotamento e queda foliar são importantes pela relação com o crescimento e a evapotranspiração (BORCHERT et al., 2002). A caracterização de padrões ecológicos, das fenofases vegetativas e reprodutivas, além da estrutura das plantas utilizadas em paisagismo são também elementos importantes para a avaliação do sucesso da implantação da espécie no ambiente.

Neste sentido, o objetivo do presente trabalho foi avaliar a fenologia vegetativa e reprodutiva da Copernicia prunifera em população natural correlacionando-a com as variáveis climáticas.

\section{MATERIAL E MÉTODOS}

\section{Área de estudo e amostragem}

O estudo foi realizado em uma população remanescente, localizada no Campus da Unidade Acadêmica Especializada em Ciências Agrárias da

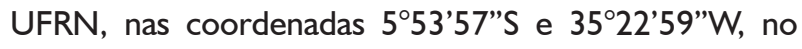

município de Macaíba, RN, considerada Área Susceptível à Desertificação (ASD), conforme lista do Programa de Ação Nacional de Combate à Desertificação e Mitigação dos Efeitos da Seca. O clima da região segundo a classificação de Köppen é uma transição entre os tipos As' e BSh', com temperaturas elevadas ao longo de todo o ano e chuvas no outono e no inverno. A matriz vegetacional da área de estudo é constituída preponderantemente por vegetação de Caatinga em diferentes subfisionomias, entre elas a Caatinga arbórea, Caatinga arbórea arbustiva e Catinga antropizada, e por áreas de encrave e de tensão ecológica com outros domínios vegetacionais, sobretudo a floresta estacional semidecidual. A vegetação local é classificada segundo - IBGE (1992), como Floresta Estacional Semidecidual de Terras Baixas, com deciduidade no final do período desfavorável atingindo mais de $80 \%$ de um dossel não muito compacto.

O local foi escolhido pelas condições aparente de preservação, sendo atribuído o valor 3, numa escala de I a 5, conforme Santos \& Vieira (2005), e com tamanho populacional adequado para as observações $(N$ $>$ 5, FOURNIER \& CHARPANTIER, 1975).

Foram selecionados, aleatoriamente, 20 indivíduos de $C$. prunifera em uma área aproximada de $600 \mathrm{~m}^{2}$. Os dados fenológicos foram obtidos entre a primeira quinzena de maio de 2010 e a primeira quinzena de dezembro de 2012, totalizando 67 observações fenológicas.

\section{Copernicia prunifera (Miller) H.E. Moore}

A carnaúba (Copernicia prunifera) é uma palmeira nativa do semiárido do Nordeste brasileiro. Apresenta distribuição principalmente nos estados de Piauí, Ceará e Rio Grande do Norte, onde ocorre em vales de rios, que muitas vezes formam extensas planícies inundáveis, resultando nos densos carnaubais (D' ALVA, 2004).

Considerada de alto valor econômico agregado e resistente à seca e inundações tão comum no Nordeste, a carnaúba é uma planta de grande porte, seu estipe pode alcançar de 10 a $20 \mathrm{~m}$ de altura e 15 a $25 \mathrm{~cm}$ de diâmetro, conservando na base os pecíolos primitivos. No ápice, estão as folhas palmadas com 0,6 a I,0 m de diâmetro e coloração verde-claro e com pecíolos medindo de I,0 a I,5 m de comprimento. As flores são inúmeras e ocorrem em panículas, que são intrafoliares e mais longas que as folhas. Os frutos, do tipo baga, são ovoides e verdes, passando a roxo-escuro à medida que amadurecem (BRAGA, 200I; LORENZI et al. 2004). É comum o uso da espécie no paisagismo local. 
A cadeia produtiva e exploração da carnaúba consistem no conjunto de atividades econômicas que utilizam o estipe, as folhas, o pecíolo, a fibra, o fruto, as raízes e principalmente a "cera-de-carnaúba", com diversas finalidades. Entretanto, com a valorização e a comercialização da cera, a tendência é o aumento da exploração desse recurso, o que pode gerar impactos nos carnaubais.

\section{Método fenológico}

Foram registradas seis fenofases: desfolhamento, enfolhamento, botão floral, floração, fruto imaturo e fruto maduro. Para a quantificação dos eventos fenológicos, foram usados o índice de atividade e o índice de intensidade de Fournier (1974). O índice de atividade refere-se à porcentagem de indivíduos manifestando o evento em cada amostragem, sendo, portanto, a presença ou ausência da fenofase específica. Esse método permite estimar a sincronia entre os indivíduos da população (BENCKE \& MORELLATO, 2002). Para a determinação do índice de intensidade de Fournier, utilizou-se uma escala intervalar semiquantitativa de cinco categorias ( 0 a 4), com intervalo de $25 \%$ entre cada categoria. Quinzenalmente, os valores obtidos de todos os indivíduos foram somados e divididos pelo valor máximo possível (número de indivíduos multiplicado por quatro). Esse valor corresponde a uma proporção que é, então, multiplicada por 100, para transformá-la em um valor porcentual (D'EÇANEVES e MORELLATO, 2004).

A

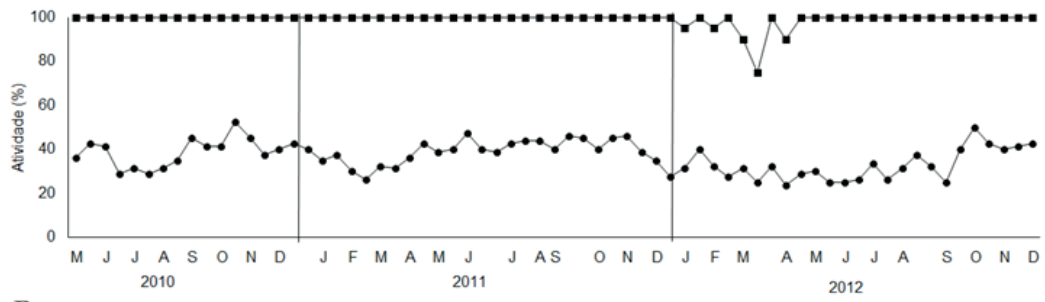

B

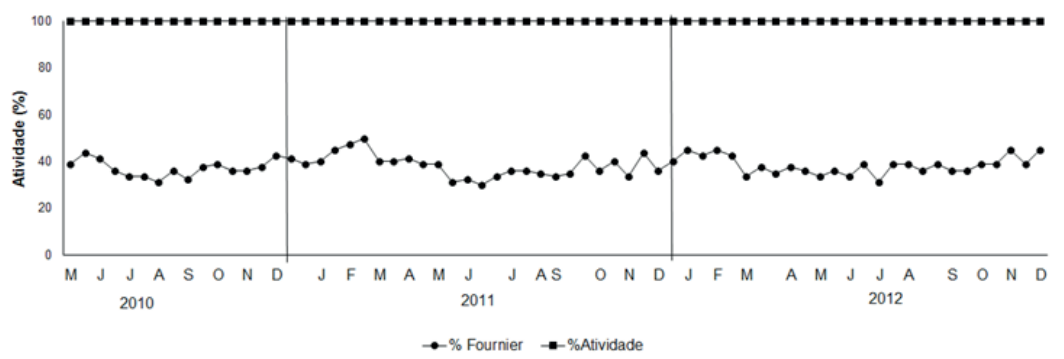

Figura I Porcentagem de enfolhamento (A) e desfolhamento (B) em uma população natural de Copernicia prunifera em Macaíba, RN. Figure I Percentage of leaf flushing (A) and leaf fall (B) in a natural population of Copernicia prunifera in Macaíba, RN. 
A fase reprodutiva da $C$. prunifera iniciou-se com a emissão de botões florais com maior intensidade nos meses de maio e junho de 2010 e 201 I (Figura 2A). No ano de 2012, observou-se menor intensidade na emissão de botões florais, em relação aos anos anteriores. $O$ maior pico de floração foi observado nos meses de junho de 2010 e 20I I (Figura2B). No ano 20I2, a presença do evento foi observada entre janeiro e agosto, porém em menor intensidade.

Devido à diversidade de padrões fenológicos entre as plantas de florestas tropicais, a classificação dos mesmos ainda é um desafio. Newstrom, Frankie \& Baker (1994), com base no florescimento de espécies da floresta tropical úmida da Costa Rica, propuseram um sistema classificatório que engloba quatro categorias: (a) contínuo, quando - florescimento é interrompido apenas por períodos esporádicos e curtos; (b) subanual, quando há vários ciclos de florescimento imprevisíveis durante o ano; (c) anual, quando há um único episódio de florescimento durante o ano; e (d) supra-anual, quando o florescimento ocorre a intervalos maiores que um ano, sendo que estes podem ocorrer regularmente ou não. A floração de Copernicia prunifera ocorre em maior atividade no início dos meses de junho e dezembro (Figura 2B), o que sugere a inclusão no grupo subanual (NEWSTROM, FRANKIE \& BAKER, 1994). Tal classificação é possível considerando o número de avaliações ( $n=67$ ) em três anos consecutivos para o presente trabalho, o que permitiu obter dados para as fenofases em diferentes situações climáticas (Figura 3). Por exemplo, a floração mais longa da Copernicia prunifera no ano de 2012 coincidiu com o ano de déficit hídrico também mais prolongado (Figura 3B), cujos valores de precipitação média nos anos de 2010, 2011 e 2012 foram: $863,7 \mathrm{~mm}$, I.289,2 mm e $790.8 \mathrm{~mm}$, respectivamente (Figura 3).

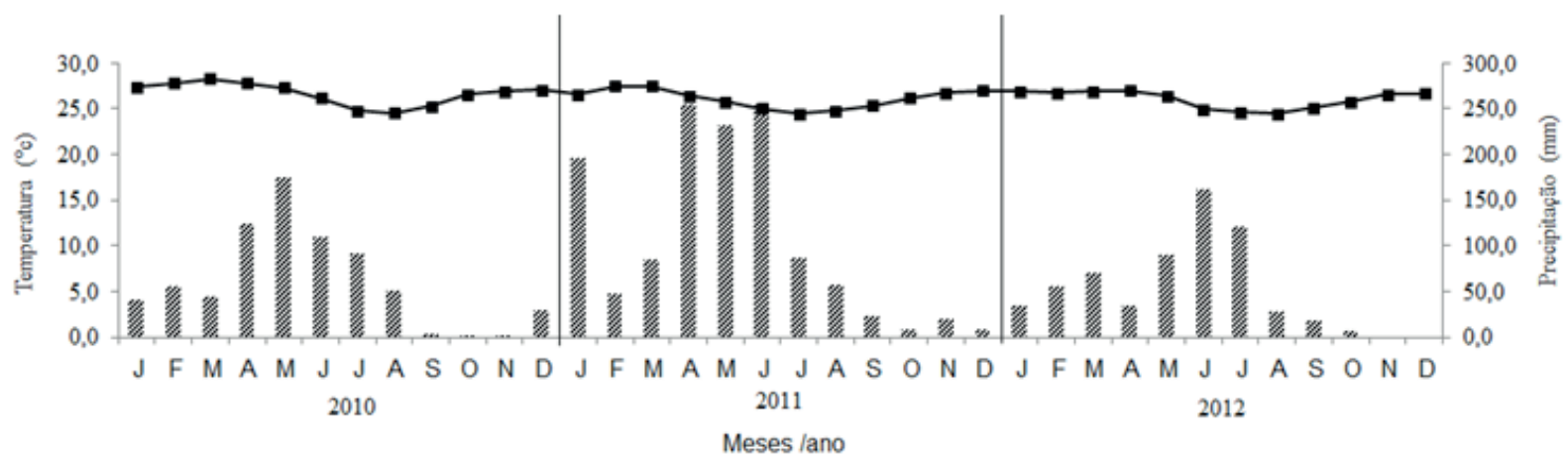

Figura 2 Valores médios mensais das variáveis climáticas de temperatura $\left({ }^{\circ} \mathrm{C}\right)$, representada pela linha, e precipitação (mm), representada pelas barras, para os três anos estudo, de 2010 a 2012. Dados obtidos no Laboratório de Variáveis Ambientais Tropicais - LAVAT, em Natal/RN.

Figure 2 Monthly mean values of climate variables of temperature $\left({ }^{\circ} \mathrm{C}\right)$, represented by line, and precipitation $(\mathrm{mm})$, represented through the bars, for the years 2010 to 2012. Data obtained in the Laboratório de Variáveis Ambientais Tropicais LAVAT, em Natal/RN.
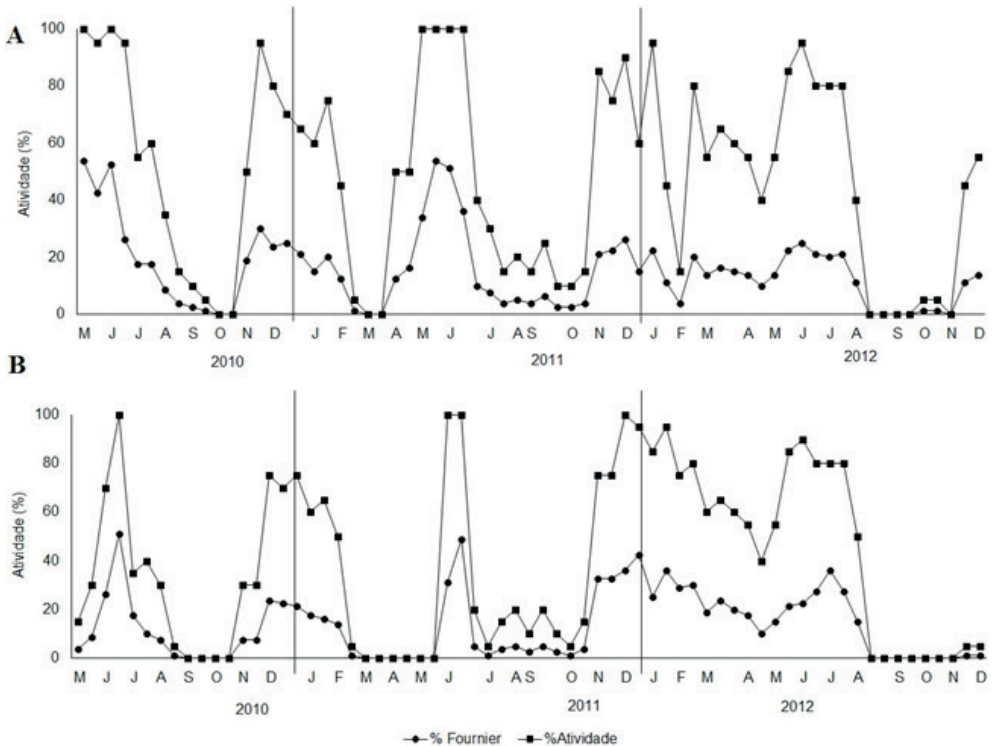

Figura 3 Porcentagem de botões florais (A) e floração (B) em uma população natural de Copernicia prunifera em Macaíba, RN.

Figure 3 Percentage of buds $(A)$ and flowering $(B)$ in a natural population of Copernicia prunifera in Macaíba, RN. 
É razoável supor que condições de stress influenciem na duração do evento de floração da espécie e consequente frutificação. De fato, observou-se menor número de indivíduos em frutificação entre os meses de junho e agosto de 2012 (Figura 4A).

A frutificação da $C$. prunifera seguiu o padrão subanual do florescimento, ou seja, ocorre mais de uma vez ao ano (Figura 4A). O período de maior intensidade de frutos imaturos foi observado nos meses de junho, julho e dezembro de 2010; janeiro, junho, julho e dezembro de $20 \mathrm{II}$. No ano de $20 \mathrm{I} 2$ seu maior pico de intensidade foi observado nos meses de janeiro e fevereiro.

Apesar dos vários eventos de frutificação ao longo do estudo, a intensidade de formação de atrasada a um dado estímulo ambiental (MARQUES et al., 2004). Isso sugere que a queda foliar está relacionada com fatores climáticos anteriores ao evento, ou seja, o aumento da temperatura, velocidade do vento e radiação e a diminuição da precipitação e da umidade relativa determinaram a perda de folhas. Desse modo, além de reduzir o número de folhas emitidas, a carnaúba provavelmente minimiza a perda de água pelo fechamento estomático e formação de uma fina camada de pó cerífero na superfície adaxial das folhas, que deve ser mais concentrada no período seco. A ausência praticamente total de chuvas e a elevação da temperatura no período de agosto a dezembro, possivelmente faz com que a planta ative mecanismos
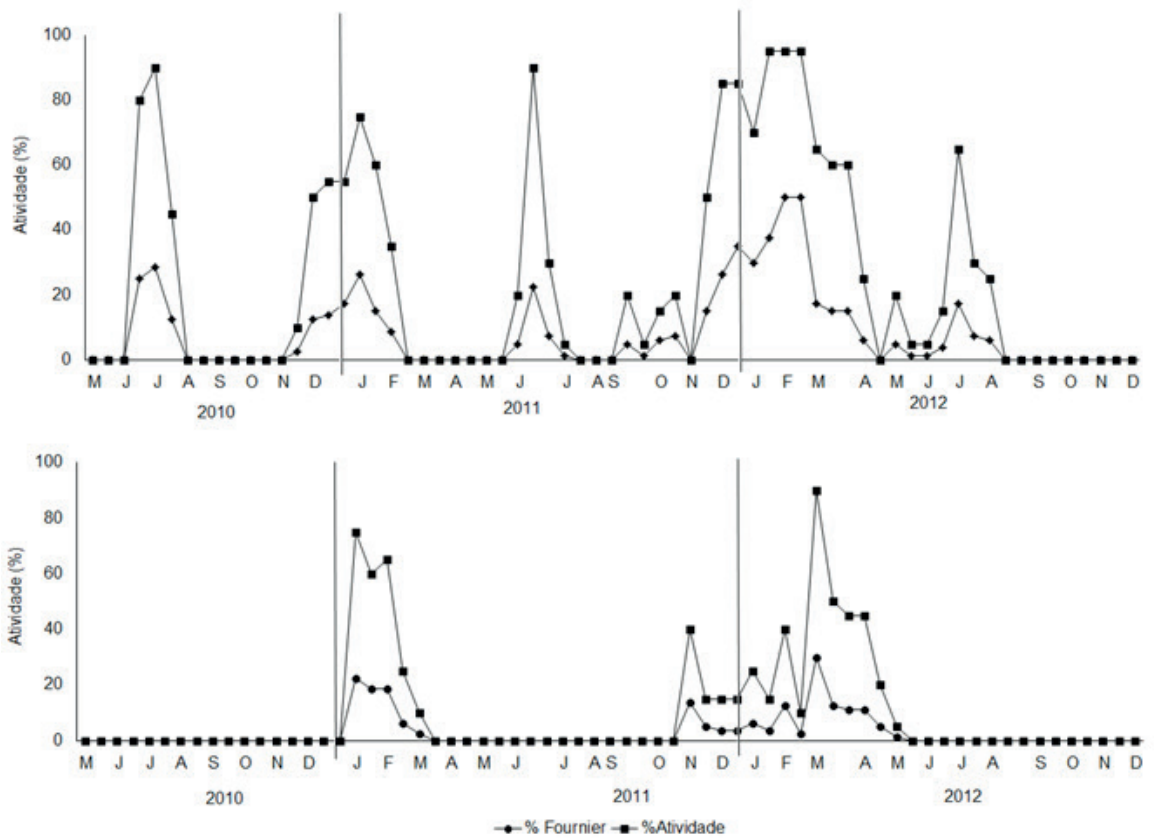

Figura 4 Porcentagem de frutos imaturos $(A)$ e frutos maduros $(B)$ em uma população natural de Copernicia prunifera em Macaíba/RN. Figure 4 Percentage of immature fruits $(A)$ and ripe fruits $(B)$ in a natural population of Copernicia prunifera in Macaíba/RN.

frutos maduros foi baixa (Figura 4B), provavelmente associada às baixas precipitações do local durante os períodos de avaliação, ou condições edafo-climáticas não favoráveis. Sabe-se que a disponibilidade de água é um fator essencial para a produção de frutos carnosos (TABARELLI; VICENTE \& BARBOSA, 2003).

\section{Correlações entre os dados fenológicos e as variáveis climáticas}

A intensidade de desfolhamento se correlacionou significativamente com as variáveis ambientais, desde as quatro quinzenas anteriores à data da ocorrência do evento (Tabela I). As plantas realmente podem apresentar uma resposta fenológica responsáveis por minimizar a perda excessiva de água por evapotranspiração, como por exemplo, o fechamento dos estômatos (TAIZ \& ZEIGER, 2006).

Estudo encontrado na literatura (REBELATTO et al., 2013), sobre a fenologia de duas espécies de ipê em área urbana, mostra que houve também correlação positiva entre a fenofase queda foliar (desfolhamento) e velocidade do vento $(r=0,8)$ para Tabebuia chrysotricha. Os autores atribuíram, em parte, à ação mecânica do vento, a qual permite o rompimento prematuro das zonas de abscisão foliares. Além disso, o estresse hídrico imposto à população analisada pode ser fator determinante (REBELATTO et al., 20l3). De fato, a diminuição da quantidade de água nas camadas mais superficiais do solo, no início da estação seca, tem sido associada com a queda 
foliar. Assim, o declínio do potencial hídrico induziria a fenofase de desfolhamento (CORLETT, 1993).

Segundo Morellato (199I), existe uma relação estreita entre queda de folhas e estacionalidade climática. Em seu estudo na Reserva de Santa Genebra/SP-Brasil, em área de floresta estacional, a deficiência hídrica foi o principal fator associado à perda de folhas. Algumas das espécies estudadas apresentaram queda de folhas mais intensa ou repetida no ano em que a estação seca foi mais rigorosa.

A fenofase de brotamento foliar (enfolhamento) apresentou correlação negativa e significativa entre a intensidade do evento e a temperatura da terceira quinzena $\left(r_{\mathrm{s} 3}=-0,247 ; \mathrm{P}=0,044\right)$ e quarta quinzena $\left(r_{s 4}=-0,28 \mathrm{I} ; \mathrm{P}=0,02 \mathrm{I}\right)$ anteriores a fenofase $\mathrm{e}$ correlação positiva e significativa entre a intensidade de enfolhamento e a velocidade do vento da primeira quinzena $\left(r_{\mathrm{Sl}}=0,325 ; \mathrm{P}=0,007\right)$ e segunda quinzena $\left(r_{s 2}=0,320 ; P=0,008\right)$ anteriores à fenofase, indicando que o enfolhamento é maior nos períodos de menor temperatura e maior velocidade do vento, sendo que esse estímulo ambiental provoca uma resposta gradual no enfolhamento da espécie (Tabela I).
A queda de folhas em espécies vegetais pode induzir em curto prazo de tempo o enfolhamento, pois reduziria a perda de água pela planta, favorecendo a reidratação dos ramos e a produção de novas folhas, mesmo em períodos secos (REICH \& BORCHERT 1984; LONGMAN \& JENIK, 1987). De fato, em muitas espécies tropicais, em diferentes biomas, é comum encontrar o episódio em que a queda foliar parece induzir o brotamento (BARBOSA et al., 1989; DUTRA, 1987; REICH \& BORCHERT, I 984; MORELLATO et al., 1 989).

O surgimento de botões florais apresentou correlação significativa com todas as variáveis climáticas analisadas. Há maior emissão de botões florais com o aumento da temperatura, da precipitação e da umidade relativa do ar. Por outro lado, há menor emissão de botões com o aumento da velocidade do vento e da radiação (Tabela 2 ).

A abertura das flores apresentou fraca relação com as variáveis ambientais, ocorrendo correlação positiva e significativa com a temperatura da terceira e quarta quinzena anteriores a fenofase e correlação negativa e significativa com a radiação da primeira e

Tabela I Correlações de Spearman $\left(r_{\mathrm{s}}\right)$ entre as médias quinzenais das variáveis climáticas e os eventos fenológicos vegetativos de Copernicia prunifera. ns, correlação não significativa $(\alpha=0,05)$; Tme, temperatura; Pre, precipitação; Ur, umidade relativa; $\mathrm{Vv}$, velocidade do vento; Rad, radiação global.

Table I Spearman's correlations $\left(r_{s}\right)$ between the averages biweekly climate variables and vegetative phenology of Copernicia prunifera. ns, not significant correlation ( $\alpha=0.05)$; Tme, temperature; Pre, rainfall; Ur, relative humidity; $V v$, wind speed; Rad, radiation global.

\begin{tabular}{|c|c|c|c|c|c|}
\hline & \multirow[t]{2}{*}{ Quinzenas } & \multicolumn{2}{|c|}{ Desfolhamento } & \multicolumn{2}{|c|}{ Enfolhamento } \\
\hline & & Atividade & Intensidade & Atividade & Intensidade \\
\hline \multirow[t]{4}{*}{ Tme } & $I^{\mathrm{a}}$ & ns & 0,553 & ns & ns \\
\hline & $2^{\mathrm{a}}$ & ns & 0,537 & ns & ns \\
\hline & $3^{a}$ & ns & 0,488 & ns & $-0,247$ \\
\hline & $4^{a}$ & ns & 0,434 & ns & $-0,281$ \\
\hline \multirow[t]{4}{*}{ Pre } & $I^{a}$ & ns & $-0,292$ & ns & ns \\
\hline & $2^{\mathrm{a}}$ & ns & $-0,324$ & ns & ns \\
\hline & $3^{\mathrm{a}}$ & ns & $-0,250$ & ns & ns \\
\hline & $4^{\mathrm{a}}$ & ns & ns & ns & ns \\
\hline \multirow[t]{4}{*}{ Ur } & $I^{a}$ & ns & $-0,37 \mid$ & ns & ns \\
\hline & $2^{\mathrm{a}}$ & ns & $-0,399$ & ns & ns \\
\hline & $3^{\mathrm{a}}$ & ns & $-0,436$ & ns & ns \\
\hline & $4^{\mathrm{a}}$ & ns & $-0,396$ & ns & ns \\
\hline \multirow[t]{4}{*}{ Vv } & $I^{\mathrm{a}}$ & ns & ns & ns & 0,325 \\
\hline & $2^{\mathrm{a}}$ & ns & ns & ns & 0,320 \\
\hline & $3^{a}$ & ns & 0,261 & ns & ns \\
\hline & $4^{\mathrm{a}}$ & ns & 0,288 & ns & ns \\
\hline \multirow[t]{4}{*}{ Rad } & $\mathrm{I}^{\mathrm{a}}$ & ns & 0,333 & ns & ns \\
\hline & $2^{\mathrm{a}}$ & ns & 0,478 & ns & ns \\
\hline & $3^{a}$ & ns & 0,494 & ns & ns \\
\hline & $4^{\mathrm{a}}$ & ns & 0,429 & ns & ns \\
\hline
\end{tabular}


Tabela 2 Correlações de Spearman $\left(r_{s}\right)$ entre as variáveis climáticas e os eventos fenológicos reprodutivos de Copernicia prunifera. ns, correlação não significativa $(\alpha=0,05)$; Tme, temperatura ; Pre, precipitação; Ur, umidade relativa; Vv, velocidade do vento; Rad, radiação global.

Table 2 Spearman's correlations $\left(r_{s}\right)$ between climate variables and reproductive phenology of Copernicia prunifera. ns, not significant correlation ( $\alpha=0.05$ ); Tme, average temperature biweekly; Pre, rainfall; Ur, relative humidity; Vv, ind speed; Rad, radiation global.

\begin{tabular}{|c|c|c|c|c|c|c|c|c|c|}
\hline & \multirow[t]{2}{*}{ Quinzenas } & \multicolumn{2}{|c|}{ Botão } & \multicolumn{2}{|c|}{ Floração } & \multicolumn{2}{|c|}{ Fruto imaturo } & \multicolumn{2}{|c|}{ Fruto maduro } \\
\hline & & Atividade & Intensidade & Atividade & Intensidade & Atividade & Intensidade & Atividade & Intensidade \\
\hline \multirow[t]{4}{*}{ Tme } & $\mathrm{I}^{\mathrm{a}}$ & 0,242 & 0,255 & ns & ns & ns & ns & 0,505 & 0,500 \\
\hline & $2^{\mathrm{a}}$ & 0,267 & 0,281 & ns & ns & ns & ns & 0,495 & 0,499 \\
\hline & $3^{a}$ & 0,407 & 0,422 & 0,308 & 0,305 & 0,253 & 0,261 & 0,457 & 0,455 \\
\hline & $4^{\mathrm{a}}$ & 0,456 & 0,468 & 0,331 & 0,324 & 0,270 & 0,276 & 0,442 & 0,440 \\
\hline \multirow[t]{4}{*}{ Pre } & $\mathrm{I}^{\mathrm{a}}$ & 0,271 & 0,249 & ns & ns & ns & ns & ns & ns \\
\hline & $2^{\mathrm{a}}$ & 0,277 & 0,253 & ns & ns & ns & ns & ns & ns \\
\hline & $3^{\mathrm{a}}$ & 0,297 & 0,283 & ns & ns & ns & ns & ns & ns \\
\hline & $4^{\mathrm{a}}$ & ns & ns & ns & ns & ns & ns & ns & ns \\
\hline \multirow[t]{4}{*}{ Ur } & $\mathrm{I}^{\mathrm{a}}$ & 0,304 & 0,292 & ns & ns & ns & ns & ns & ns \\
\hline & $2^{\mathrm{a}}$ & 0,283 & 0,273 & ns & ns & ns & ns & ns & ns \\
\hline & $3^{a}$ & ns & ns & ns & ns & ns & ns & ns & $-0,24 I$ \\
\hline & $4^{\mathrm{a}}$ & ns & ns & ns & ns & ns & ns & $-0,296$ & $-0,299$ \\
\hline \multirow[t]{4}{*}{$\mathrm{Vv}$} & $\mathrm{I}^{\mathrm{a}}$ & $-0,310$ & $-0,304$ & ns & ns & ns & ns & ns & ns \\
\hline & $2^{\mathrm{a}}$ & $-0,318$ & $-0,313$ & ns & ns & ns & ns & ns & ns \\
\hline & $3^{a}$ & $-0,262$ & $-0,245$ & ns & ns & ns & ns & ns & ns \\
\hline & $4^{a}$ & ns & ns & ns & ns & ns & ns & ns & ns \\
\hline \multirow[t]{4}{*}{ Rad } & $\mathrm{I}^{\mathrm{a}}$ & $-0,425$ & $-0,395$ & $-0,284$ & $-0,266$ & ns & ns & ns & ns \\
\hline & $2^{a}$ & $-0,356$ & $-0,329$ & $-0,255$ & $-0,243$ & ns & ns & ns & ns \\
\hline & $3^{a}$ & ns & ns & ns & ns & ns & ns & ns & ns \\
\hline & $4^{a}$ & ns & ns & ns & ns & ns & ns & ns & ns \\
\hline
\end{tabular}

segunda quinzena anteriores a fenofase tanto para 0 índice de atividade quanto para o índice de intensidade, indicando que a floração ocorre no período de maior temperatura e menor radiação (Tabela 2).

As relações entre os frutos imaturos e o período de maior temperatura são indicadas pelas correlações positivas entre esse evento e a variável temperatura com atraso de três a quatro quinzenas do evento fenológico (Tabela 2). Frutos maduros apresentaram correlação negativa e significativa com a umidade relativa na quarta quinzena anterior à ocorrência do evento e correlação positiva e significativa com a temperatura nas quatro quinzenas anteriores à fenofase, evidenciando na população estudada maior número de árvores com frutos maduros em períodos com elevada temperatura.

Durante o período de observações, foi verificada baixa produção de frutos maduros e consequentemente baixa dispersão das sementes. Conforme Miller (2002), a baixa produção de frutos pode ser resultado de diversos fatores, como reduzida taxa de polinização, ocorrência de aborto do fruto imaturo, além da baixa fertilidade do solo. Neste sentido, além do déficit hídrico no local de estudo, a baixa frequência de polinização e provável aborto de frutos imaturos são aspectos que devem ser investigados nos próximos estudos com C. prunifera. $\mathrm{O}$ fato de nem todos os indivíduos terem florescido e a ocorrência de baixa frutificação, podem estar relacionados ainda às condições do local de estabelecimento do vegetal, pois além das características intrínsecas das espécies, as variações climáticas também influenciam a fenologia (FERRAZ et al., 1999). Por último, ressalta-se que número de flores produzidas por um vegetal não sugere necessariamente a quantidade de frutos imaturos que serão observados (ADLER et al. 1998). De maneira semelhante, a quantidade de frutos imaturos produzidos por uma infrutescência pode não resultar na mesma quantidade de frutos maduros por causa dos fatores diversos discutidos acima.

Ferraz etal. (1999) constataram que a frutificação nas espécies de Cordia ecalyculata, Croton floribundus e Cedrela fissilis está relacionada com a precipitação e que a influência do clima é complexa, pois cada variável 
climática parece ter um peso diferente na fenologia das espécies. De fato, a ocorrência e intensidade de uma determinada fenofase geralmente estão associadas a um ou vários fatores abióticos atuando em conjunto, como temperatura, precipitação, umidade, disponibilidade de nutrientes, e aos fatores bióticos, como atividade de polinizadores e dispersores, comportamento de predadores de sementes e herbívoros (VILELA et al., 2008; NAZARENO \& REIS, 20I2). Todos esses fatores, por sua vez, estão sujeitos a interferências das mudanças no ambiente físico (JANZEN, 1980). De acordo com as respostas às mudanças ambientais, as espécies ou indivíduos estão sujeitos à seleção natural, dessa maneira respondendo diferentemente a um mesmo estímulo (CHAPMAN et al., 2005).

Assim, há necessidade de realizarem-se acompanhamentos fenológicos de longa duração, para que possa avaliar a maior ou menor interferência de fatores bióticos e abióticos nos padrões fenológicos apresentados ao longo de vários ciclos reprodutivos.

\section{CONCLUSÕES}

Os indivíduos de Copernicia prunifera têm os eventos vegetativos assincrônicos, com perda de folhas e emissão de folhas novas durante o ano. A floração e frutificação é subanual, com dois períodos no ano. A formação dos frutos e dispersão ocorre em períodos de maiores temperaturas e menor umidade relativa do ar. Entretanto, há baixa quantidade de frutos maduros em anos com menor precipitação. Este estudo contribui para o conhecimento de aspectos básicos da ecologia das populações naturais de $C$. prunifera até então desconhecidos.

\section{REFERÊNCIAS}

ADLER, G. H. Impacts of resource abundance on populations of a tropical forest rodent. Ecology, v. 79, p. 242-254, 1998.

AYRES, M., AYRES Jr, M., AYRES, D. L., SANTOS, A. A. S. Bioestat 5.0 aplicações estatísticas nas áreas das ciências biológicas e médicas. Belém: IDSM, 2007. 364p.

BARBOSA, D. C.; ALVES, J. L. A.; PRAZERES S. M. PAIVA, A. M. A. Dados fenológicos de 10 espécies arbóreas de uma área de caatinga (Alagoinha-PE) Acta Botânica Brasilica, v. 3, p. 109-117, 1989.

BENCKE, C. S. C.; MORELLATO, L. P. C. Comparação de dois métodos de avaliação da fenologia de plantas, sua interpretação e representação. Revista Brasileira de Botânica, v. 25, n. 3, p. 269-276, 2002.
BORCHERT, R.; RIVERA, G.; HAGNAUER, W. Modification of vegetative phenology in a tropical semi-deciduous forest by abnormal drought and rain. Biotropica, v. 34 , p. 27-39, 2002.

BRAGA, R. Plantas do Nordeste, especialmente do Ceará. Coleção Mossoroense, 5.ed. 496p, 2001.

CAMARGO-RICALDE, S. L.; DHILLION, S. S.; GARCÍA, V. Phenology, and seed production and germination of seven endemic Mimosa species (Fabaceae-Mimosoideae) of the Tehuacán-Cuicatlán Valley, Mexico. Journal of Arid Environments, v. 58, p. 423-437, 2004.

CHAPMAN, C. A; CHAPMAN, L. J; STRUHSAKER, T. T; ZANNE, A. E; CLARK, C. J.; POULSEN, J. R. A long-term evaluation of fruiting phenology: importance of climate change. Journal of Tropical Ecology, v. 21, p. I-I4, 2005.

CORLETT, R. T. Reproductive phenology of Hong Kong shrubland. Journal of Tropical Ecology, v. 9, n . 4, v. 50 I-5I0, 1993.

D’ALVA, O. A. O Extrativismo da Carnaúba no Ceará. Fortaleza, CE, 2004

D'EÇA-NEVES, F. F; MORELLATO, L. P. C. Métodos de amostragem e avaliação utilizados em estudos fenológicos de florestas tropicais. Acta Botanica Brasilica, v. 18, n. I, p. 99-108, 2004.

DUTRA, R.C. Fenologia de dez espécies arbóreas nativas do Cerrado de Brasília, DF. Brasil Florestal, v. 62, p. 23-4I, 1987.

FERRAZ, D. K.; ARTES, R.; MANTOVANI, W.; MAGALHÃES, L. M. Fenologia de árvores em fragmento de mata em São Paulo, SP. Brazilian Journal of Biology, v. 59, n. 2, p. 305-317, 1999.

FOURNIER, L. A. Un método cuantitativo para la medición de características fenológicas em árboles. Turrialba, v. 24, n. 4, 1974.

FOURNIER, L. A. \& CHARPANTIER, C. El tamaño de la muestra y la frecuencia de las observaciones en el estudio de las características fenológicas de los árboles tropicales. Turrialba, v. 25, p. 45-48, 1975.

HALL, P.; WALKER, S.; BAWA, K.S. Effect of forest fragmentation on genetic diversity and mating system in a tropical tree, Pithecellobium elegans. Conservation Biology, v. 10, n. 3, p. 757-768, 1996.

IBGE - Manual técnico da vegetação brasileira. Fundação Instituto Brasileiro de Geografia e Estatística. Rio de Janeiro. 1992.

JANZEN, D. H. Ecologia vegetal nos trópicos. EPU e EDUSP. São Paulo. 1980. 
LORENZI, H.; SOUZA. H. M. Palmeiras brasileiras e exóticas cultivadas. Nova Odessa: Instituto Plantarun, v. I, 2004, 4I6p.

LONGMAN, K. A.;.J.JENIK. Tropical forest and its envioronment. Logman Singapore Publishers, Singapore. 1987.

MARQUES, M. C. M.; ROPER, J. J.; SALVALAGGIO, A. P. B. Phenological patterns among plant life forms in a Subtropical Forest in Southern Brazil. Plant Ecology, v. I73, p. 203-2।3. 2004.

MORELLATO, L. P. C. Estudo da fenologia de árvores, arbustos e lianas de uma floresta semidecídua no sudeste do Brasil. Tese de Doutorado, Universidade de Campinas, Campinas, São Paulo. 1991.

MORELLATO, L. P. C.; RODRIGUES, R. R.; LEITÃO-FILHO, H. F; JOLY, C. A. Estudo comparativo da fenologia de espécies arbóreas de floresta de altitude e floresta mesófila semidecídua na Serra do Japi, Jundiaí, São Paulo. Revista Brasileira de Botânica, v. 12, p. 85-98, 1989.

NAZARENO, A. G.; REIS, M. S. Linking phenology to mating system: exploring the reproductive biology of the threatened palm species Butia eriospatha. Journal of Heredity, v. I03, p. 842-852. 2012.

NEWSTROM, L. E.; FRANKIE, G. W.; BAKER, H. G. A new classificacion for plant phenology based on flowering patterns in lowland tropical rain forest trees at La Selva, Costa Rica. Biotropica, Kansas, v. 26, n. 2, p. I4|-159, 1994.

MILLER, C. Fruit production of the ungurahua palm (Oenocarpus bataua subsp. Bataua, Arecaceae) in an indigenous managed reserve. Economic Botany, v. 56, n. 2, 165-176, 2002.
REICH, P. B. \& R. BORCHERT. Changes with leaf age in stomatal function and water status of several tropical tree species. Biotropica, v. 20, p. 60-69, 1988.

REBELATTO, D.; LEAL, T. S.; MORAES, C. P. Fenologia de duas espécies de ipê em área urbana do município de Araras, São Paulo, Brasil. Revista da Sociedade Brasileira de Arborização Urbana, Piracicaba, v. I, n. 8, p. I-16, 2013.

SANTOS, R. M.; VIEIRA, F. A. Análise estrutural do componente arbóreo de três áreas de cerrado em diferentes estádios de conservação no município de Três Marias, Minas Gerais, Brasil. Cerne, Lavras, v. II, n. 4, p. 399-408, 2005.

TABARELLI, M.; VICENTE, A.; BARBOSA, D. C. A. Variation of seed dispersal spectrum of woody plants across a rainfall gradient in northeastern Brazil. Journal of Arid Environmental, v. 53, p. 197-210, 2003.

TAIZ, L.; ZEIGER, E. Fisiologia vegetal. 3.ed. Porto Alegre: Artmed, 2004. 719 p.

VIEIRA, F. A.; CARVALHO, D. Maturação e morfometria dos frutos de Miconia albicans (Swartz) Triana (Melastomataceae) em um remanescente de floresta estacional semidecídua montana em Lavras, MG. Revista Árvore, v. 33, p. 1015-1023. 2009.

VILELA, G. F.; CARVALHO D.; VIEIRA F. A. Fenologia de Caryocar brasiliense Camb. (Caryocaraceae) no Alto Rio Grande, sul de Minas Gerais. Cerne, v. I4, n. 4, p. 317-329, 2008. 
